\title{
Active Gripper for Hot Melt Joining of Micro Components
}

\author{
Sven Rathmann, Annika Raatz, and Jürgen Hesselbach \\ Institute of Machine Tools and Production Engineering, \\ Technical University Braunschweig, \\ Langer Kamp 19b, 38106 Braunschweig, Germany \\ s.rathmannatu-bs.de
}

\begin{abstract}
Precision assembly of hybrid micro systems requires not only a high precision handling and adjusting of the parts but also a highly accurate and fast bonding technique. In this field adhesive technology is one of the major joining techniques. At the Collaboration Research Center 516, a batch process based on a joining technique using hot melt adhesives was developed. This technique allows the coating of micro components with hot melt in a batch. The coating process is followed by the joining process. Due to this, the time between coating and joining can be designed variably. Because of the short set times of hot melt adhesives short joining times are possible. For this assembly process adapted heat management and adapted gripper systems are very important. Primarily, the conception and construction of suitable grippers, which realize the complex requirements of the heat management, meet a high challenge. This paper presents an active gripper system for the joining of micro parts using hot melt adhesives. Furthermore, first examinations and results of an assembly process using this gripper for bonding with hot melt adhesives are presented.
\end{abstract}

Keywords: Precision joining operations, hot melt adhesives, active gripper systems.

\section{Introduction}

The necessity of integrating more and more functions into micro components by unchanging or decreasing components' size makes a high precision assembly in the sub micron area more and more important [1]. To reach this goal, many endeavors in the field of high accurate handling and positioning systems were and are made [2, 3, 4]. Another very important part is the use of a suitable bonding technology. The most important technology is the adhesive bonding technique [5]. Requirements to this bonding technique are: a rapid fast curing time, the possibility of a pre-application of the adhesive, a low or nonexistent shrink of the adhesive and the possibility to use this bonding technique in batch processes. Nowadays, chemical or light reacting adhesives are used. These adhesives normally achieve high strength of the bond. But the disadvantages of these adhesives are their long curing times (light reacting adhesives have short curing times, less than 10 seconds, but it is not always possible to irradiate the bonding area), the constraint to apply the adhesive directly or shortly before the joining process and their low suitability for batch processes. The approach developed in 
the Collaborative Research Center 516 "Design and Manufacturing of Active Microsystems" is the use of hot melt adhesives. The main advantages are extremely short set cycles, the possibility of pre-applying the adhesive and the time-delayed joining procedure [6]. A disadvantage is their low adhesion. The comparison of the advantages and disadvantages shows that this bonding technology is an interesting alternative for the assembly of hybrid micro systems.

To establish this technology, adhesive and application technologies as well as the assembly process have to be developed. Furthermore, the process parameters must be determined. A very important aspect for the process design is the kind of heating technology that should be used. Therefore, special process components, such as fixtures and grippers as well as the heat management concepts should be developed. In [7] two approaches to solve these problems are described. The following section discusses these concepts and augments the approaches using a phase model. The assembly process will also be described. In the third section a new active gripper concept will be presented. It can be used for joining micro components with hot melt adhesives. Finally, first results of test assemblies will be shown and discussed.

\section{Adapted Assembly Strategies for Hot Melt Joining}

Heat management is an integral part of the selection and the design of an assembly process using hot melt adhesives, as the volume of the hot melt is quite small and the thermal capacity is rather low. This results in the hot melt not being able to store much thermal energy. Thus heating the hot melt adhesive directly is not recommendable. On the contrary the thermal capacity of the component and the gripping system is much higher. Hence the characteristics of the grippers and the components such as thermal capacity and thermal conductivity are much more influential to the heat management. To achieve this, special considerations have to be taken in account when designing a specific assembly process, as the choice of a suitable heating source is crucial for process design. For heating up the gripper unit there are multiple devices available: heating plates, infrared heaters, lasers, heating foils, heating ceramics and Peltier elements, or a combination of these.

In general, the concepts of heat management can be split into a passive and an active concept [8]. The passive heat management concept makes use of the principle of heat storage to supply the energy for the joining process. The active concept carries a heat source inside the gripper system. Depending on the design, the heat emission has to be controlled in order to customize the heating to specific needs of the process.

The solid line in Figure 1 indicates a temperature profile of the passive heat management concept. The joining sequence is divided into three consecutive phases: a heating phase, a processing phase and a post-processing phase. During the first phase, the heating phase, the gripper and the component are heated by a heat source to the working temperature $\mathrm{T}_{\mathrm{Hp}}$. The handling, e.g. movement of the component from the heat source towards the target position, is carried out in the processing phase, which is limited by $t_{\mathrm{Hp}}$ and $\mathrm{t}_{\mathrm{Kp}}$. When the component touches the substrate the post-processing phase begins. The temperature drops substantially, the hot melt sets and reaches its final strength. 
In contrast to the passive heat management concept, the heat source of the active heat management concept (dashed line in Figure 2) is integrated into the gripping or clamping unit. With this setup heat can be continuously transmitted into the component during the handling or the joining process. Thus the temperature can be controlled and kept at a constant level during the processing phase or heating can instantly be applied when necessary. The working temperature is thereby dependent on the melting temperature and not on the process time. This is why the processing temperature can be set at a lower level.

The advantage of the active heat management concept is the independence of the assembly process from the heat capacity of components which allows flexibility in the process design.

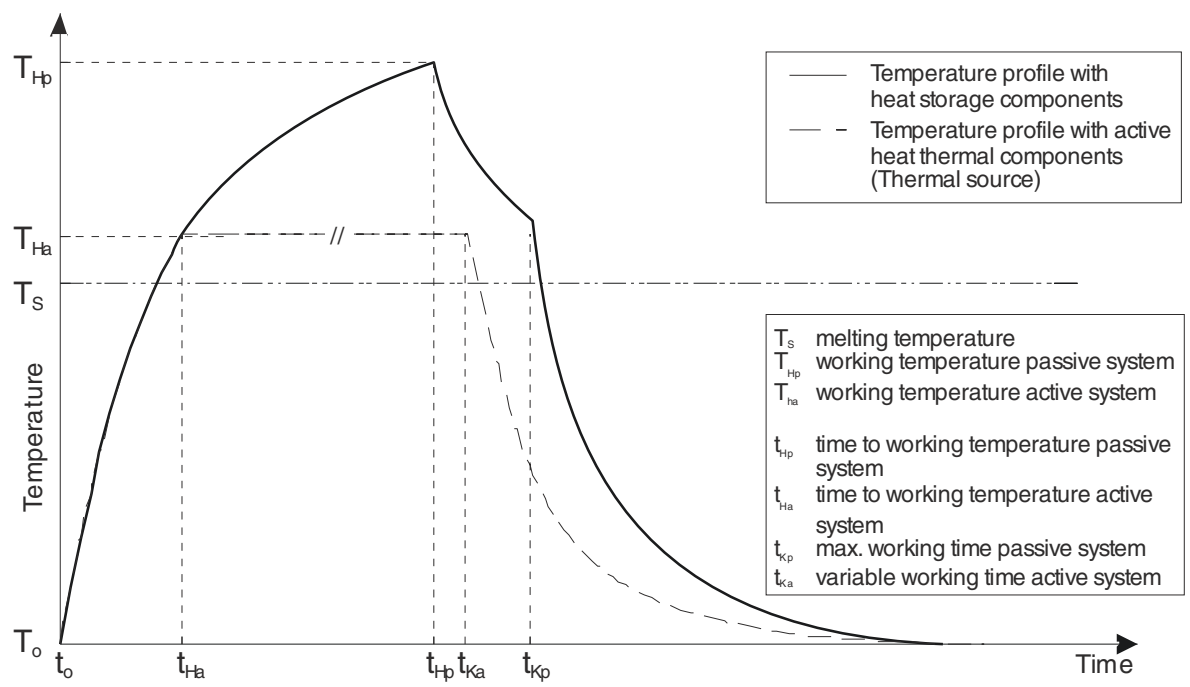

Fig. 1. Temperature profiles on different heat management concepts

Consequently, the assembly of micro components has to accomplish the following steps to follow the described heat management concept:

1. gripping the hot melt coated component

2. heating up the component (heating phase)

3. aligning the component with the joining position (processing phase)

4. joining (post-processing phase)

5. wait until the component temperature has dropped below the melting temperature (post-processing phase)

6. release the bonded component

In the following examinations and experiments the hot melt adhesive "Vestamelt 732" will be used. This adhesive has its melting temperature $\mathrm{T}_{\mathrm{S}}$ in the area of 100 to $110{ }^{\circ} \mathrm{C}$. Experiences with this adhesive show that a working temperature $\mathrm{T}_{\mathrm{Hp}}$ of about $140{ }^{\circ} \mathrm{C}$ is suitable for joining with this adhesive. 


\section{Active Gripper System}

Figure 2 shows the active gripper concept. It consists of a heat source, which is carried out as Peltier element, a heat sink, a vacuum gripper and an isolation to the robot system. The bottom side of the gripper will be called primary side. This side is important for the heat exchange to the component. The side of the Peltier element which is connected to the heat sink will be called secondary side.

Peltier elements are heat pumps. Depending on the impressed current direction the Peltier element generates a heat flux from one to the other side of the element. Thus a temperature difference between the sides will be generated. Normally, Peltier elements will be used as thermoelectric cooler. With standard Peltier elements a maximum temperature difference of $70{ }^{\circ} \mathrm{C}$ can be achieved. Using Peltier elements as heat source a higher temperature difference is possible. This is due to the effect that the internal heat losses are added to the generated heat flux . Without a suitable control Peltier elements can be heated up to their destruction.

Due to the dimension of the handled parts and the philosophy of size adapted handling systems [2] the gripping system should not be bigger than $3 \mathrm{~cm}$ in all directions. The used Peltier element has a dimension of $16 \times 16 \mathrm{~mm}^{2}$ and a power of $34 \mathrm{~W}$. With heat sink, isolation, sensors and connection parts the whole dimension of the gripper is $35 \times 15 \times 30 \mathrm{~mm}^{3}$.

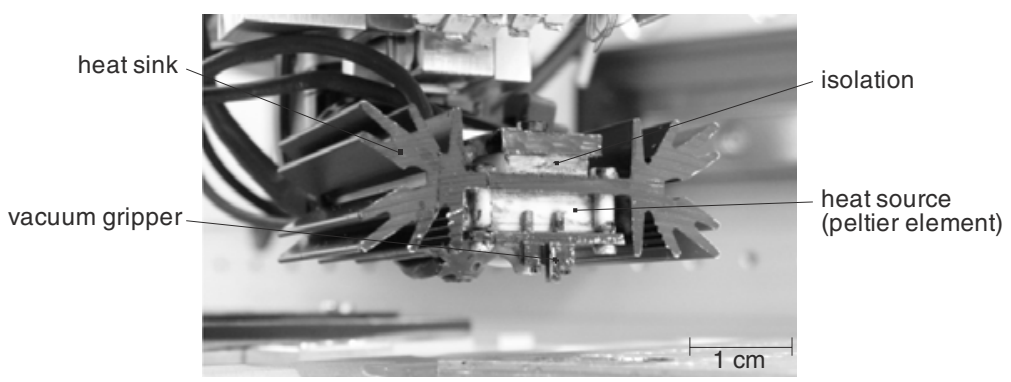

Fig. 2. Active gripper system

In the heating mode of the active gripper the heat flux will be generated from the secondary to the primary side. Therefore, a fast heating of the gripped component and the coated adhesive will be done. The gripper is controlled by a bang-bang controller. Due to the heat inertia this simple control concept reaches a suitable quality of control of $\pm 2.5^{\circ} \mathrm{C}$. The parameters of the controller are selected in such a way that a mean gripper temperature of about $140{ }^{\circ} \mathrm{C}$ is reached. Thus the working temperature $\mathrm{T}_{\mathrm{Hp}}$ of about $130{ }^{\circ} \mathrm{C}$ on the bottom side of the component adjusts itself.

By switching the current direction to the second mode of the gripper system, the cooling mode can be used. In this mode the heat flux from the primary to the secondary side will be generated. With sufficient heat flow at the heat sink a fast cooling effect on the primary side can be achieved. Thus it is possible to reach further reductions of the joining time. 


\section{Experiments}

\subsection{Assembly Process and Parameters}

For the experiments and the validation of the active gripper system a size adapted assembly system was used. Fig. 3 shows the assembly system. The robot (micabo-f2) provides four degrees of freedom, for part handling and an additional degree for focusing the integrated 3D-vision sensor. The workspace is $160 \times 400 \times 15 \mathrm{~mm} 3$ and thus enables flexible assembly of various micro systems. Therefore, two assembly fixtures of size 4, according to DIN 32561, could be prepositioned in the clamping device. The micabo-f2 has a repeatability of 0.6 micrometers with a standard deviation of $3 \sigma$ as measured according to EN ISO 9283 [9]. The standard control of the assembly system was enhanced for the control of the active gripper. Thus a fully automatic assembly process is possible.

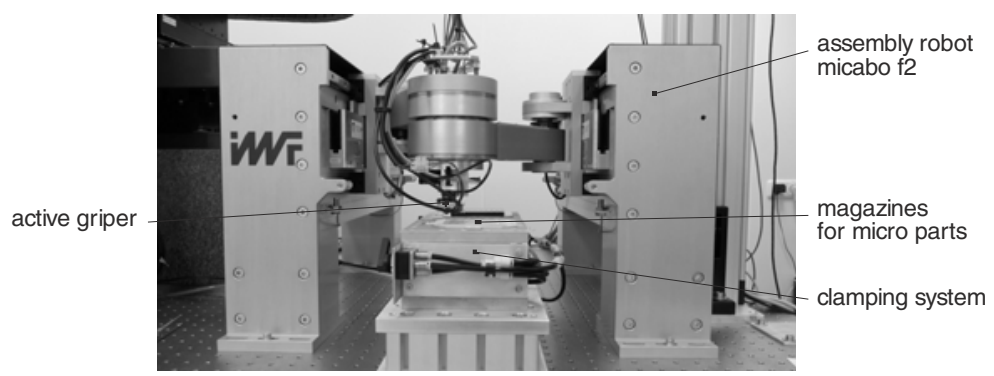

Fig. 3. Assembly robot micabo f2 (IWF Braunschweig)

To detect possible shifts of the components during the joining process a continuously detection of the components with the integrated 3D-vision system is possible. Furthermore the joining force can be measured with an integrated 3D force sensor. In Fig. 3 the test components are also shown. During the assembly process the smaller part $\left(3 \times 2 \mathrm{~mm}^{2}\right)$ will be gripped and joined with the bigger part $\left(8 \times 4 \mathrm{~mm}^{2}\right)$. On the bottom site of the smaller part the hot melt adhesive is pre-applied in form of dots with a diameter of ca. $500 \mu \mathrm{m}$ and a height of ca. $100 \mu \mathrm{m}$. The assembly process was implemented as described in section 2.

Fig. 4 shows the temperature profile of an exemplary assembly process. The temperature curves of the primary and of the secondary side are displayed. Marked are the three phases of the heat management.

The curves show an assembly process starting with a cold gripper, whereby the duration of the heating phase is about $21 \mathrm{~s}$. If the gripper is in a stabile working state, the time for heating can be reduced to about $12 \mathrm{~s}$ (Fig. 5). The process phase takes about $10 \mathrm{~s}$. In this phase the alignment and the joining process takes place. The components will be aligned with a maximum positioning error of $1 \mu \mathrm{m}$. The positioning correction will be done with a lock and move algorithm. During the joining process, the assembly system adjusts the components with a gap of about $50 \mu \mathrm{m}$ between the components. The joining force was nearly constant during the test assemblies and was in the 


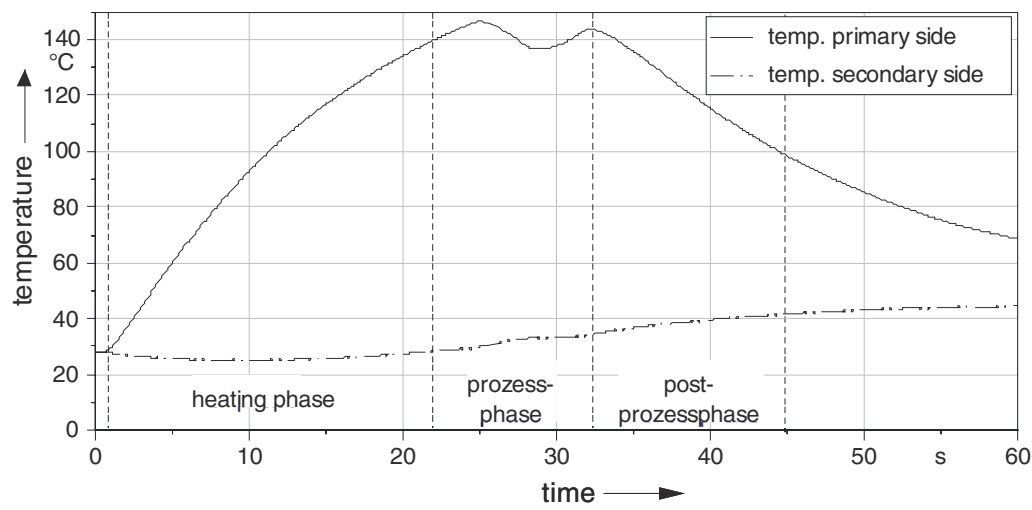

Fig. 4. Temperature profile of an assembly process

area of about $1 \mathrm{~N}$. During the post-process phase the temperature of the primary side drops below $100{ }^{\circ} \mathrm{C}$ the within about $12 \mathrm{~s}$ and the hat melt adhesive sets. After the post-process phase the part can be released. The total assembly process takes less than $50 \mathrm{~s}$ which is much faster than joining processes with chemical reacting adhesives.

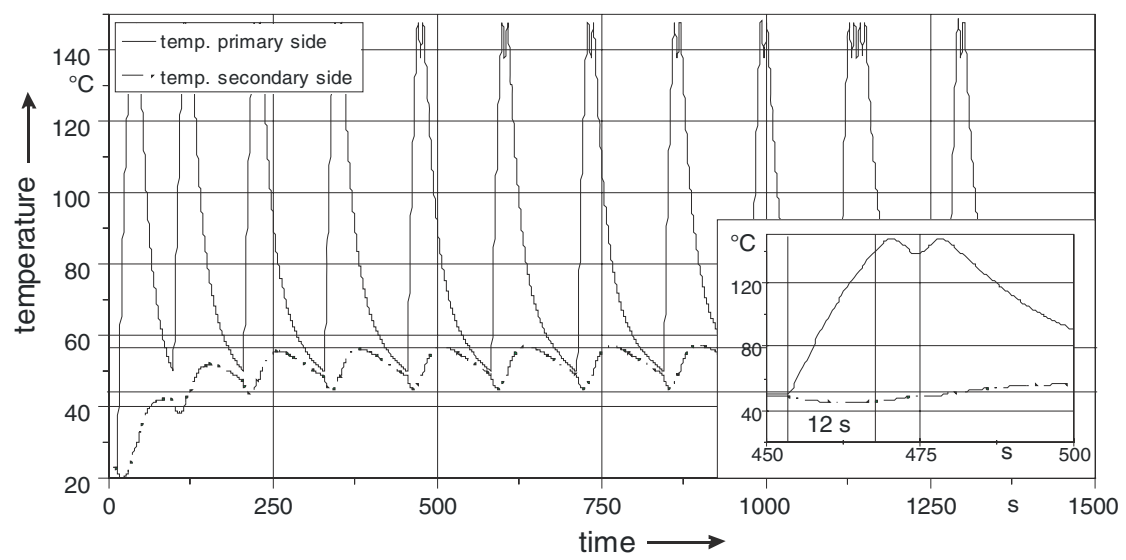

Fig. 5. Assembly cycles with stable temperature state of the secondary side

During a typical assembly processes many single assembly steps will take place. Since in these processes there are usually no long waiting times between the assembly steps, a quasi stable temperature state above the room temperature of the secondary side will be reached (Figure 5). This temperature depends on the thermal parameters of the gripping system and the parameters of the assembly process. At a process time of $10 \mathrm{~s}$ and a cooling temperature of the primary side of about $50{ }^{\circ} \mathrm{C}$ a process cycle time of about $120 \mathrm{~s}$ can be reached. In this case the temperature of the secondary side lies in the range of 45 to $55^{\circ} \mathrm{C}$. 


\subsection{Assembly Results and Discussion}

The accuracy of assembly is an important criterion for the assessment of a newly developed assembly process. Therefore, assembly examinations with the parts described in section 4.1 were done and the uncertainty of assembly was measured. To evaluate the effect of the joining technology, the positioning uncertainty before joining and the assembly uncertainty after joining were measured. However, the obtained deviation described not only the effect of the joining processes but also the influence of the handing systems. This could be a lateral displacement of the part at the surface of the vacuum gripper and also a displacement in the magazine.

The uncertainty of assembly of the described assembly process was $11.9 \mu \mathrm{m}$. Less the position uncertainty of $1.4 \mu \mathrm{m}$ the portion of the joining process and the handling components to the assembly uncertainty is $10.5 \mu \mathrm{m}$. Compared to the accuracy of assembly process using the passive heat management $(69 \mu \mathrm{m}$ [10]) a reduction of the uncertainty of $85 \%$ using the active heat management could be reached.

A great part of the joining uncertainty can be ascribed to the big size variation of the adhesive dots. Furthermore, the accuracy of the positioning in z-direction depends on the variation of the component height and the measuring of the $z$ - distance between the components resulted from the 3D-vision sensor.
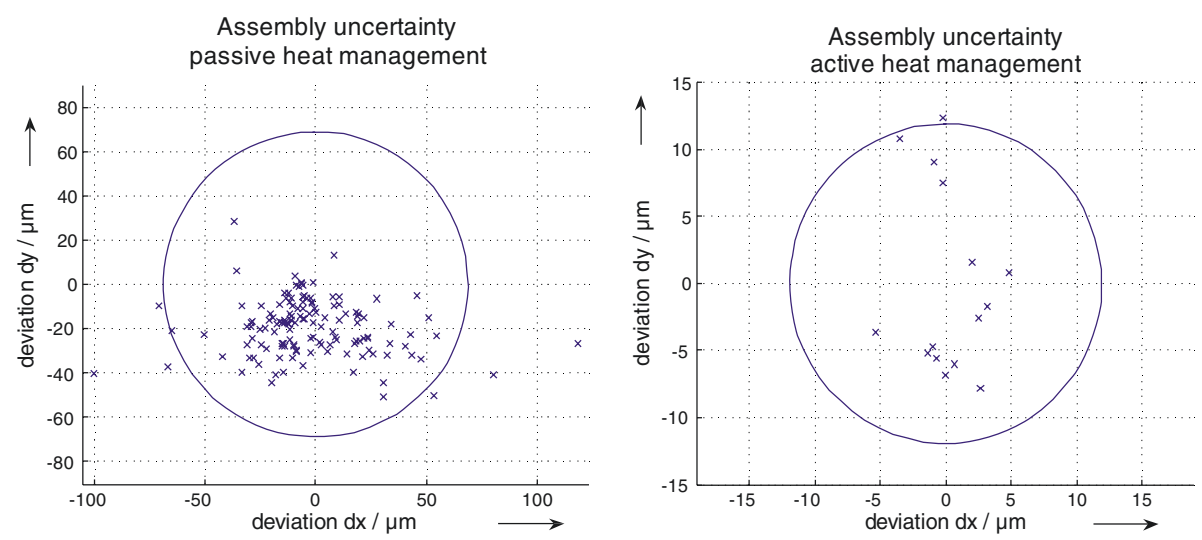

Fig. 6. Uncertainty of the assembly process (left: passive management, right: active management)

\section{Conclusion and Outlook}

In this paper an alternative joining process for hybrid micro systems based on hot melt adhesives was presented. Based on the outcomes of the passive heat management and associated gripper systems, a gripper system for the active heat management was presented. The heat source of the gripper based on the Peltier effect and can be used as cooling element also. The assembly examinations show the functionality of the active gripper system and offer good results for the assembly accuracy. Further increase of the accuracy can be reached by improving the holding force of the gripper, 
the placement accuracy in z-direction and optimizing the application process of the hot melt dots. To reduce the process time of the assembly process the active cooling effect of the gripper can be used. In this field further investigations have to be done.

Acknowledgments. The authors gratefully acknowledge the funding of the reported work by the German Research Center (Collaborative Research Center 516).

\section{References}

1. Bauer, G., et al.: Flexibles Montagesystem für die Feinwerk- und Mikrotechnik. Maschinenmarkt 105, 30-35 (1999)

2. Schöttler, K., Raatz, A., Hesselbach, J.: Precision Assembly of Active Microsystems with a Size-Adapted Assembly System. Micro-Assembly Technologies and Applications, 199206 (2008) ISBN 978-0387-77402-2

3. Gaugel, T., Bengel, M., Malthan, D.: Bulding a Mini-Assembly System from a Technology Construction Kit. In: Proc. of International Precision Assembly Seminar (IPAS), Bad Hofgastein, Astria, March 17-19 (2003)

4. Clavel, R., et al.: High Precision Parallel Robots for Micro-Factory Applications. In: Robotic Systems for Handling and Assembly, pp. 285-296 (2005)

5. Zäh, M.F., Schilp, M., Jacob, D.: Kapsel und Tropfen - Fluidauftrag für Mikrosysteme. Evolutionäre und revolutionäre Verfahren in der Dispenstechnik. Wt-Werkstattstechnik online 92(9), 428-431 (2002)

6. Böhm, S., et al.: Micro Bonding using hot melt adhesives. Journal of Adhesion and Interface. The Society of Adhesion \& Interface 7(4), 28-31 (2006)

7. Rathmann, S., Raatz, A., Hesselbach, J.: Concepts for Hybrid Micro Assembly using Hot Melt Joining. Micro-Assembly Technologies and Applications, 161-169 (2008) ISBN 978-0387-77402-2

8. Rathmann, S., et al.: Strategies for the Use of Hot Melt Adhesives for Highly Accurate Micro Assembly. In: Proceeding of the 2nd CIRP Conference on Assembly Technologies \& Systems, Toronto, Canada, September 21-23, pp. 407-416 (2008)

9. Simnofske, M., Schöttler, K., Hesselbach, J.: micaboF2 - Robot for Micro Assembly. Production Engineering, XII(2), 215-218 (2005)

10. Rathmann, S., et al.: Sensor-guided micro assembly of active micro systems by using a hot melt based joining technology. Microsystem Technologies 14(12), 1975-1981 (2008) 